\title{
Osteomielitis crónica multifocal recurrente: estado del arte
}

\section{Chronic recurrent multifocal osteomyelitis: State of the art / Osteomielite crônica multifocal recorrente: estado da arte}

Fecha de recibido: 22 de febrero de 2016

Fecha de aprobación: 04 de abril de 2016
Forma de citar este artículo: Gallego HD, VelásquezFranco CJ, Mesa MA Osteomielitis crónica multifocal recurrente: estado del arte. Med U.P.B 2016:35(1): 32-40.

DOI:10.18566/medupb.v35n1.a05

1. Residente de Ortopedia y Traumatología. Universidad Pontificia Bolivariana. Medellín, Colombia

2. Grupo UNIR. Escuela de Ciencias de la Salud. Universidad Pontificia Bolivariana. Medellín, Colombia

3. Servicio de Reumatología. Clínica Universitaria Bolivariana. Medellín, Colombia

Dirección de correspondencia: Carlos Jaime Velásquez Franco. Correo electrónico: carjaivel@hotmail.com
Hernán Darío Gallego Eusse ${ }^{1}$, Carlos Jaime Velásquez-Franco ${ }^{2,3}$, Miguel Antonio Mesa Navas $^{2,3}$

\section{RESUMEN}

La osteomielitis crónica multifocal recurrente es una enfermedad autoinflamatoria que se caracteriza por focos estériles de inflamación ósea que producen dolor recurrente. Es una entidad poco frecuente que tiene como manifestaciones cardinales la presencia de lesiones óseas inflamatorias en las regiones metaepifisiarias de los huesos largos que predomina en miembros inferiores. Esta enfermedad, con frecuencia asociada con síntomas sistémicos y lesiones en piel, hace parte del grupo de enfermedades que conforma el síndrome: artritis-dermatitis. Los hallazgos radiológicos más comunes son las áreas de osteolisis rodeadas de un halo esclerótico delgado, sin reacción perióstica asociada o la formación de secuestros óseos. La resonancia nuclear magnética permite hacer una detección precoz de la entidad y facilita su diagnóstico diferencial, principalmente, con infecciones y tumores óseos. El tratamiento está basado en la información obtenida mediante reportes de caso y series de pacientes y consiste en el uso de antiinflamatorios no esteroideos y en casos refractarios, bisfosfonatos y antagonistas del factor de necrosis tumoral alfa. El pronóstico de esta enfermedad, en general, es bueno, con un curso clínico benigno y autolimitado, en la mayoría de los casos.

Palabras clave: osteomielitis; inflamación; hiperostosis.

\section{ABSTRACT}

Chronic recurrent multifocal osteomyelitis is a sterile autoinflammatory disease characterized by foci of sterile inflammation in the bone that generate recurrent pain. It is a rare entity in which the cardinal clinical manifestation is the presence of inflammatory bone lesions in metaepiphyseal regions of long bones, predominating in lower limbs. The disease is often associated with systemic symptoms and skin involvement making it part of the arthritis-dermatitis syndrome. The most common radiographic finding is osteolysis surrounded by a thin sclerotic halo, without periosteal reaction associated with bone formation or bone sequestrum. Magnetic resonance imaging allows early detection and facilitates the differential diagnosis between osteomyelitis and infections or bone tumors. Treatment is supported mainly by case reports and series of patients and consists of the use of nonsteroidal anti-inflammatory drugs and the use of bisphosphonates or tumor necrosis factor alpha antagonists in refractory cases. The prognosis of this disease is generally good, with a benign and self-limited clinical course in most cases.

Keywords: osteomyelitis; inflammation; hyperostosis.

\section{RESUMO}

A osteomielite crônica multifocal recorrente é uma doença autoinflamatória que se caracteriza por focos estéreis de inflamação óssea que produzem dor recorrente. É uma 
entidade pouco frequente que tem como manifestações cardinais a presença de lesões ósseas inflamatórias nas regiões metaepifisárias dos ossos longos que predomina em membros inferiores. Esta doença, com frequência associada com sintomas sistémicos e lesões na pele, faz parte do grupo de doenças que conforma o síndrome: artritedermatite. As descobertas radiológicos mais comuns são as áreas de osteólise rodeadas de um rasto esclerótico delgado, sem reação periosteal associada ou a formação de sequestros ósseos. A ressonância nuclear magnética permite fazer uma deteç̧ão precoce da entidade e facilita seu diagnóstico diferencial, principalmente, com infecções e tumores ósseos. O tratamento está baseado na informação obtida mediante reportes de caso e séries de pacientes e consiste no uso de antinflamatórios não esteróides e em casos refratários, bisfosfonatos e antagonistas do fator de necrose tumoral alfa. O prognóstico desta doença, em geral, é bom, com um curso clínico benigno e autolimitado, na maioria dos casos.

Palavras chave: osteomielite; inflamação; hiperostose.

\section{INTRODUCCIÓN}

\section{Definición y desarrollo histórico}

La osteomielitis crónica multifocal recurrente (CRMO, por sus siglas en inglés) se puede catalogar como un desorden autoinflamatorio que se caracteriza por la presencia de múltiples focos estériles de inflamación en el hueso que desencadenan episodios de inflamación y dolor, generalmente autolimitados, pero con potencial para recurrir con el transcurso de meses $o$ años ${ }^{1-4}$.

La primera descripción de esta entidad fue realizada por Giedon hacia 1972, cuando reportó cuatro casos de niños manejados con diagnósticos de osteomielitis subaguda y crónica que presentaban múltiples lesiones simétricas que afectan los huesos largos y adyacentes a las placas de crecimiento, en los que el resultado de los cultivos de hueso fue negativo para bacterias y con un resultado histológico que reportaba infiltrado celular plasmático ${ }^{5,6}$. En 1978, Björksten et al. Introdujeron el término de CRMO y reportaron una serie de nueve pacientes con edades entre cuatro a 26 años que tenían historia de inicio insidioso de fiebre, edema local y dolor en diferentes regiones óseas. Los hallazgos radiológicos sugerían osteomielitis, con lesiones en la clavícula y regiones metafisiarias de los huesos largos. El curso clínico se caracterizaba por periodos in- termitentes de exacerbación y mejoría en el transcurso de varios años; las biopsias óseas mostraban cambios inflamatorios no específicos con infiltración granulocítica $\mathrm{y}$ fueron negativos los cultivos de hueso $\mathrm{y}$ hemocultivos para bacterias y hongos ${ }^{7}$.

En 1987 se acuñó el nombre de síndrome Sapho (sinovitis, acné, pustulosis, hiperostosis, osteítis) por autores franceses para agrupar una serie de entidades clínicas y radiológicas constituidas por lesiones específicas de la piel, huesos y articulaciones. Para muchos, la CRMO es el equivalente pediátrico del Sapho, pero, a la fecha, hay controversia respecto a si se trata de una misma entidad presente en diferentes grupos de edad o si son distintos finales de un mismo espectro clínico ${ }^{8-11}$.

\section{Epidemiología}

La CRMO es una patología relativamente rara, con una prevalencia estimada de 1/1 000000 casos; sin embargo, si se considera dentro del espectro del síndrome Sapho, se podría hablar de una incidencia real de alrededor de 1/10 000. Es por esto que algunos autores creen que se trata de una entidad subdiagnosticada, cuya frecuencia seguramente es mayor. Por lo general, afecta a niños y adolescentes, con una edad promedio de inicio de 10 años y predominantemente en el género femenino, aunque también hay reportes de adultos jóvenes. Puede haber una historia 
familiar de enfermedades inflamatorias, pero únicamente en el 6\% habrá un miembro con el diagnóstico de $\mathrm{CRMO}^{1,2,4,12}$.

\section{Etiopatogenia}

La causa exacta de esta entidad continúa siendo un misterio, aunque los autores proponen diversas teorías a partir de su presentación clínica y asociación con otras patologías. La primera hipótesis es la etiología infecciosa, para algunos razonable, si se considera la frecuente asociación de la CRMO con lesiones dermatológicas (acné grave). Los autores que están en favor de esta teoría parten de la base de que Propionibacterium acnes ha demostrado jugar un papel fundamental en la etiopatogenia del Sapho, en parte, por la relación directa de esta bacteria con el desarrollo de acné, manifestación cutánea cardinal de este síndrome. Assmann et al. reportaron, en 2009, un estudio en el que, a una cohorte de 30 pacientes con dicho diagnóstico, se le trató durante 16 semanas con antibióticos (azitromicina, clindamicina o doxiciclina), se logra remisión clínica de las lesiones óseas y cutáneas; sin embargo, luego de tres meses de suspendida la terapia se presentaron recaídas en 12 sujetos que completaron el seguimiento y no se encontraron diferencias en las escalas de actividad de las lesiones óseas y dermatológicas entre las semanas una y $28^{13}$. Se discute entonces si realmente el beneficio que se logró se debió al efecto antimicrobiano o al efecto antiinflamatorio de la terapia antibiótica (azitromicina, principalmente). Por otro lado, a la fecha, no se han logrado demostrar aislamientos microbiológicos de las áreas comprometidas, a pesar del avance de las técnicas moleculares, por lo que el hecho de que la CRMO se deba a una infección por patógenos de bajo grado continúa siendo un tópico controversial ${ }^{13-15}$.

La segunda hipótesis es la autoinflamatoria, que parte del hecho de que, en el curso natural de la enfermedad, se presentan exacerbaciones espontáneas de inflamación, la ausencia de títulos altos de autoanticuerpos, el predominio de neutrófilos en las lesiones tempranas y la susceptibilidad genética. Estudios recientes sugieren que hay alteración en la producción de citoquinas proinflamatorias, con una respuesta atenuada de la señal extracelular regulada por kinasas, que disminuye la expresión de $\mathrm{Sp}-1$, un factor de transcripción que, a su vez, induce la interleuquina 10 (IL-10), lo que lleva a niveles disminuidos de esta última y a niveles aumentados de otras citoquinas denominadas proinflamatorias, como: IL-6, IL-8, IL-18 y factor de necrosis tumoral alfa (TNF-a) que perpetúan las manifestaciones clínicas y explican, además, la leucocitosis y elevación de los reactantes de fase aguda encontrada ocasionalmente en los pacientes con CRMO o Sapho ${ }^{1,8}$.
La tercera hipótesis parte de una base autoinmune. Grosjean et al, en un grupo de 90 pacientes con diagnóstico de Sapho, encontró anticuerpos antinucleares (ANA) en $15.5 \%$, anticuerpos antitiroideos en $3.3 \%$ y anti-Ro en $2.3 \%$, mientras que los anti-DNA, anticuerpos anticitrulina y antimitocondriales fueron negativos en todos los pacientes ${ }^{16}$. Si bien los autores sugieren una relación con la autoinmunidad con base en estos resultados, los títulos tomados para considerar ANA positivo fue de 1/160, pero la baja prevalencia de antígenos específicos como Ro $y$ anti DNA cuestionan la relevancia de este resultado $y$, más, cuando la hipótesis principal era la presencia de una mayor proporción de anticuerpos antitiroideos en esta población. Esta hipótesis fue descartada por los autores. En conclusión, no existe evidencia sólida para considerar la CRMO como una enfermedad autoinmune.

\section{Cuadro clínico}

La CRMO se presenta con episodios recurrentes e insidiosos de dolor óseo inflamatorio y cambios locales sobre las áreas afectadas (edema, eritema y calor). Por las características recurrentes del cuadro, debe recordarse que los síntomas pueden presentarse en los mismos sitios previamente afectados o comprometer áreas diferentes. Estos episodios suceden de forma espontánea, aunque, en algunos pocos casos, hay un evento traumático que precede el inicio de la sintomatología. El compromiso sistémico con síntomas como fiebre de bajo grado, malestar, pérdida de peso, entre otros, ocurre en aproximadamente una tercera parte de los pacientes y muchas veces orientará hacia otros diagnósticos diferenciales ${ }^{1-4,8,12,15,17-19}$. Las lesiones son comúnmente multifocales y simétricas, aunque algunos pacientes presentan lesiones únicas; el promedio del número de lesiones es entre dos y trescuatro durante el curso de la enfermedad. Cualquier hueso es susceptible de resultar comprometido, pero, generalmente, la afección se observa en las áreas metaepifisiarias de los huesos largos de los miembros inferiores, en el siguiente orden de frecuencia: tibia distal, tibia proximal, pelvis, fémur proximal, fémur distal y calcáneo. Aunque el compromiso de la clavícula, el esternón y la mandíbula es menos frecuente, estas lesiones son altamente sugestivas de CRMO. El compromiso vertebral genera, primordialmente, vértebras planas y fracturas por compresión y varía entre 4\%-30\% de los casos s, $^{1,20-24}$.

En el subgrupo de pacientes catalogados como Sapho, se presentan discretas variaciones de acuerdo con la edad. Así, la pared anterior del tórax (articulación esternocostoclavicular) es el sitio más comúnmente afectado en adultos; mientras que el tercio medio de la clavícula es el sitio de mayor afectación en la población pediátrica. Por su parte, el esqueleto axial es el segundo sitio más 
comprometido en ambos grupos, entre 32\%-52\% de los casos y el orden de compromiso en este segmento se da en la columna torácica, lumbar y cervical ${ }^{8,9}$.

Alrededor de 30\% de los pacientes con CRMO tiene manifestaciones extraóseas adicionales, con una fuerte asociación con desórdenes inflamatorios de la piel, como: pustulosis palmoplantar (20\%-50\%), acné, psoriasis vulgar, pioderma gangrenoso y dermatomiositis e, igualmente, desórdenes inflamatorios del tracto intestinal, como enfermedad de Crohn y colitis ulcerativa ${ }^{1,7,25,26}$.

\section{Diagnóstico}

Se requiere un alto índice de sospecha para el diagnóstico de la CRMO, con base en datos de la historia clínica en relación con las características, localización del dolor y síntomas asociados, datos del examen físico como lesiones asociadas en la piel, signos inflamatorios locales, hallazgos imaginológicos sugestivos y resultados de laboratorio para descartar infección y, sobre todo, para que, durante los periodos de exacerbación, se pueda encontrar una elevación discreta o moderada de los reactantes de fase aguda. El clínico enfrenta tres escenarios para el diagnóstico de la CRMO: (i) el primero es relativamente fácil: se encuentran lesiones óseas en los sitios típicos, hallazgos característicos en la piel e imágenes radiológicas patognomónicas que se mencionarán a continuación; (ii) el segundo es un escenario difícil: las lesiones óseas se presentan en sitios menos comunes y no hay manifestaciones en piel; (iii) en el tercer caso el diagnóstico clínico es imposible: únicamente habrá una lesión ósea sin signos característicos de la entidad y es menester un alto índice de sospecha y la toma de biopsia para estudio histológico para el diagnóstico definitivo ${ }^{8}$.

Entre los pocos criterios clasificatorios para el diagnóstico de osteítis no bacteriana se encuentra el de Jansson, quien plantea unos criterios mayores que incluyen compromiso multifocal, psoriasis o pustulosis palmo-plantar, lesiones óseas radiológicas osteolíticas/escleróticas y una biopsia de hueso estéril con signos de inflamación o fibrosis. Los criterios menores incluyen curso mayor a seis meses, asociación con enfermedades autoinmunes (excluidas psoriasis o pustulosis palmoplantar), hiperostosis y leve elevación de la proteína $\mathrm{C}$ reactiva o la velocidad de sedimentación globular. El diagnóstico se establece si hay dos criterios mayores o un criterio mayor y, por lo menos, tres criterios menores ${ }^{27}$.

Adicionalmente, se habla de algunos criterios que predicen de mejor manera una osteítis no bacteriana como: temperatura corporal normal; lesiones óseas simétricas; compromiso vertebral, clavicular o esternal; lesiones radiológicas con esclerosis marginal y PCR mayor de 1 $\mathrm{mg} / \mathrm{dL}$. Estos criterios alcanzan, para algunos autores, un valor predictivo positivo de $97 \%$ y una sensibilidad de $68 \%{ }^{28}$.

\section{Estudios con imágenes}

Las radiografías simples constituyen el examen inicial en cualquier niño o adulto con dolor óseo y sospecha de CRMO. La descripción radiológica inicial, hecha por Giedon, consiste en lesiones osteolíticas inmediatamente adyacentes a la placa de crecimiento de los huesos largos que, durante la evolución, cicatrizan con marcada esclerosis ${ }^{5}$.

De esa forma, los hallazgos descritos en relación con una CRMO consisten en una osteolisis que puede o no estar rodeada por un halo esclerótico delgado, observada típicamente en el área metafisiaria y contigua a la fisis que, en ocasiones, se extiende y compromete la diáfisis con engrosamiento cortical, sin reacción en el periostio, asociada con la formación de secuestros óseos que es imposible diferenciar desde el punto de vista radiológico, de infecciones o tumores. En el periodo temprano de cicatrización se observa esclerosis en el mismo sitio o una mezcla de lesiones osteolíticas y escleróticas, pero, generalmente, en el transcurso de dos años, el hueso recupera su apariencia radiológica normal. Las radiografías también pueden ser normales en estadios tempranos de la enfermedad, por lo que se debe mantener un alto índice de sospecha ${ }^{1-5,7,10,29-33}$.

$\mathrm{Si}$ se considera el compromiso multifocal característico de esta patología, es preciso esperar múltiples lesiones óseas y solicitar radiografías de todos los sitios en los que haya dolor ${ }^{32,33}$.

El compromiso de la clavícula inicia en el tercio medial y progresa de manera gradual hasta comprometer el tercio externo. Los cambios radiológicos son divididos en tres estadios: (i) el primero comprende el área de los ligamentos costoclaviculares y se visualiza edema de los tejidos blandos, erosiones o nuevas formaciones óseas; (ii) el estadio II representa un compromiso generalizado de la región esternocostoclavicular con aumento de esclerosis en el tercio medio de la clavícula, la primera costilla y el área adyacente del esternón; (iii) el último estadio consiste en un aumento de la osteoesclerosis, con hipertrofia del tercio medio de la clavícula y potencial anquilosis de las articulaciones adyacentes ${ }^{32,33}$.

Los cambios radiológicos en el esqueleto axial incluyen lesiones de las esquinas vertebrales, espondilodisquitis no específica, lesiones osteodestructivas y osteoescleróticas, osificación paravertebral y sacroiliítis; por este motivo, en ocasiones, la CRMO se confunde con las espondiloartritis ${ }^{32,33}$.

Respecto a otros estudios con imágenes, la resonancia nuclear magnética (RNM) representa una herramienta 
útil para guiar el diagnóstico y realizar el seguimiento de pacientes con CRMO, con una sensibilidad mayor a la de la radiografía convencional, la posibilidad de realizar una detección temprana al observar edema medular o inflamación de los tejidos blandos adyacentes y monitorizar la progresión de la enfermedad para diferenciar entre lesiones óseas crónicas que muestran una baja intensidad de señal en secuencias T1 y T2 o lesiones activas que aparecen hipointensas en $\mathrm{T} 1$ e hiperintensas en $\mathrm{T} 2^{34,35}$.

La RNM corporal total está descrita como el examen más sensible para detectar las lesiones múltiples de CRMO, a la vez que permite el estudio simultáneo de la anatomía ósea, los tejidos blandos e identificar el compromiso de la epífisis. No obstante, su alto costo, la falta de disponibilidad en todos los centros hospitalarios, el tiempo prolongado para su realización (alrededor de 45 minutos) y la necesidad de sedación en la población pediátrica, limitan su uso rutinario ${ }^{36}$.

La gamagrafía ósea es útil en la detección de lesiones silentes; sin embargo, a veces, la interpretación es difícil porque se llegan a confundir lesiones óseas activas y denominarlas como placas de crecimiento normales. La gamagrafía requiere alta exposición a radiación ${ }^{37}$.

\section{Biopsia y estudio histológico}

Dentro de los criterios diagnósticos se incluye una biopsia ósea abierta o guiada por imágenes, particularmente, en el caso de pacientes con una lesión ósea solitaria que amerite descartar diagnósticos diferenciales como, por ejemplo, malignidad. En otros escenarios en los que los hallazgos clínicos y radiológicos que orienten claramente a CRMO no habría necesidad de realizarla ${ }^{38}$.

Histológicamente pueden coexistir lesiones agudas, subagudas o crónicas y los hallazgos iniciales son osteolisis e infiltración por neutrófilos y células gigantes, posteriormente, aparecen linfocitos, células plasmáticas y monocitos. La esclerosis e hiperostosis ocurren en etapas $\operatorname{tardías}^{38}$.

\section{Diagnósticos diferenciales}

Diversas patologías deben ser incluidas en el diagnóstico diferencial de la CRMO (Tabla 1); sin embargo, la infección y los tumores óseos son los que representan mayor preocupación.

La osteomielitis aguda hematógena con compromiso de múltiples huesos llega a ser común en la infancia, por lo que deben realizarse una anamnesis y un examen físico, además, solicitar todos los paraclínicos para descartar un compromiso infeccioso. En relación con los tumores óseos deben sospecharse, particularmente, en pacientes con lesiones únicas, síntomas asociados como fatiga, pérdida de peso, sudoración nocturna y hallazgos como linfadenopatías, hepatoesplenomegalia y citopenias; como se mencionó previamente, en este escenario, la biopsia es fundamental para el diagnóstico y la clasificación ${ }^{39}$.

\section{Síndrome Sapho y CRMO}

Como se mencionó anteriormente, el síndrome Sapho es una enfermedad rara que afecta predominantemente niños y adultos menores de 60 años y que consiste en una serie de patologías cutáneas y óseas que se presentan de manera simultánea. En los adultos, el sitio más comúnmente comprometido es la pared anterior del tórax (65\%-90\% de los casos), seguida por la columna vertebral (30\%), mientras que, en los niños, los huesos largos constituyen el sitio más afectado. Las manifestaciones musculoesqueléticas más comunes incluyen: oligoartritis erosiva o no erosiva de rodillas, tobillos, articulaciones metacarpofalángicas y metatarsofalángicas.

Tabla 1. Diagnósticos diferenciales de la osteomielitis crónica multifocal recurrente.

\begin{tabular}{lcc}
\hline Infecciones & Tumores & Otras condiciones \\
\hline Osteomielitis bacteriana & Osteoma osteoide & Artritis idiopática juvenil \\
Tuberculosis & Osteoblastoma & Artritis psoriásica \\
Brucelosis & Osteosarcoma & Osteoporosis \\
Salmonelosis & Rabdomiosarcoma & Fractura ósea \\
& Sarcoma de Ewing & Sd de Majeed \\
& Neuroblastoma & Sd de deficiencia del antagonista del \\
& Leucemia & receptor de IL-1 \\
& Linfoma & Cherubismo
\end{tabular}


$\mathrm{La}$ artritis tiene un inicio insidioso y se asocia con rigidez matinal. Las lesiones de la piel consisten en: acné severo (conglobata o fulminans) y pustulosis palmo-plantar en aproximadamente $50 \%-75 \%$ de los casos y pueden preceder, presentarse simultáneamente o años después de las lesiones óseas ${ }^{40}$.

Algunos autores consideran que la presencia de CRMO, aun en ausencia de manifestaciones cutáneas, es suficiente para establecer el diagnóstico de síndrome Sapho, mientras que otros la consideran el equivalente pediátrico de este mismo síndrome. La controversia continúa respecto a si se trata de una misma entidad o patologías independientes. La CRMO exhibe todas las características esenciales del Sapho, incluidas: osteítis, hiperostosis y la asociación con desórdenes de la piel y, por su parte, el síndrome Sapho está asociado con enfermedades inflamatorias intestinales al igual que la $\mathrm{CRMO}^{41}$. Por otro lado, existen ciertas diferencias entre ambas entidades, que se expresan en la Tabla 2.

Los criterios diagnósticos también son similares para ambas entidades, excluidos la cronicidad y el compromiso multifocal para el Sapho ${ }^{40,41}$.

Según esto, la tendencia actual es la de considerar la CRMO como una enfermedad dentro del espectro del Sapho que afecta predominantemente a los niños y, por ello, el tratamiento y pronóstico serán comunes.

\section{Tratamiento}

Los objetivos del tratamiento en los sujetos con CRMO son: el alivio de los síntomas, promover la curación ósea, evitar la deformidad y prevenir nuevos episodios. El método óptimo para lograrlo se desconoce y, con respecto a los medicamentos que se disponen actualmente, persisten preguntas sobre los efectos a largo plazo asociados con su uso crónico, principalmente en niños ${ }^{1}$.

La gravedad de la enfermedad, el grado de inflamación y las enfermedades concomitantes son los factores que determinarán el momento de inicio y el tipo de tratamiento.

La terapia física es un pilar relevante porque permitirá mantener rangos de movimientos funcionales de las diferentes articulaciones, evitar contracturas e incrementar la fuerza muscular. Los anti-inflamatorios no esteroideos
(Aines) representan la primera opción terapéutica, con efectividad en el control del dolor en alrededor de $80 \%$ de los individuos ${ }^{1}$. En el contexto de artritis o compromiso vertebral, los Aines han demostrado no ser tan efectivos y se debería considerar otra estrategia. Los esteroides también son comúnmente citados como beneficiosos en el manejo de pacientes con CRMO, pero hay pocos reportes en la literatura que respalden su uso y no se tiene un consenso respecto a la dosis apropiada y duración del tratamiento en una población particularmente susceptible a los efectos secundarios de estos fármacos por su uso continuo, tal como el cierre temprano de las placas de crecimiento $^{42}$.

Los bisfosfonatos, como medicamentos que inhiben la resorción ósea, han demostrado ser efectivos en el tratamiento de la CRMO, especialmente indicados en pacientes con marcada esclerosis ósea, niños con compromiso de columna vertebral, mandíbula o incremento de captación en las últimas fases de la gamagrafía ${ }^{43}$. Pamidronato ha sido el más frecuentemente usado en diferentes regímenes reportados en la literatura, en infusiones semanales (días 1, 7, 14, 28 y 56) o infusiones de $60 \mathrm{mg}$ durante tres días consecutivos y luego cada tres meses ${ }^{44,45}$.

Igualmente, los antagonistas del factor de necrosis tumoral (anti-TNF) han demostrado ser efectivos para el manejo de esta patología, con unas indicaciones precisas en pacientes con marcada inflamación (dolor inflamatorio grave, fiebre, elevación de la PCR), incremento de la captación en fases tempranas de la gamagrafía y en enfermedad refractaria a la terapia con Aines o bisfosfonatos. A la fecha no hay ningún consenso de cuál sea el medicamento anti-TNF ideal ${ }^{46}$.

Otros fármacos han sido reportados de forma anecdótica en casos refractarios como metotrexate, sulfasalazina, colchicina e interferón, pero no hay indicaciones precisas y falta evidencia que soporte su uso en estos sujetos ${ }^{47-49}$.

Respecto al uso de antibioticoterapia, azitromicina en especial, ya se mencionó previamente el estudio de Assmann et al., en el que se demostró un beneficio transitorio de este tratamiento, pero con posterior recaída de la sintomatología, por lo que, a la fecha, no hay ningún beneficio demostrado que justifique el tratamiento rutinario con antibióticos ${ }^{13}$.

Tabla 2. Principales diferencias entre el síndrome Sapho y la osteomielitis crónica multifocal recurrente.

\begin{tabular}{lcc}
\hline Enfermedad & Sitios esqueléticos comprometidos & Población afectada \\
\hline CRMO & Metáfisis de huesos largos & Pediátrica \\
Síndrome Sapho & Esqueleto axial y pared anterior del \\
tórax & Adultos jóvenes \\
\hline
\end{tabular}


Se han realizado procedimientos quirúrgicos, como decorticación o resección parcial/completa del área afectada del hueso, en algunas series aisladas de pacientes, pero generalmente no están recomendados por la alta tasa de recurrencia. No obstante, en casos de una grave limitación funcional o preocupaciones estéticas, como cuando hay afectación de la mandíbula, son una opción a considerar ${ }^{8}$.

\section{Pronóstico}

La CRMO es considerada, en términos generales, una patología benigna y autolimitada. La historia natural está caracterizada por remisiones prolongadas con periodos de exacerbación, aunque sin mayores secuelas. Sin embargo, estudios recientes han demostrado que a una media de seguimiento de 10 años, aproximadamente 25\%-59\% de los individuos permanecen con la enfermedad activa desde el punto de vista de imágenes, aun encontrándose asintomáticos. La duración de esta actividad se ha relacionado directamente con el número de lesiones e inversamente con la edad de inicio. En la Tabla 3 se mencionan los predictores de enfermedad crónica que se han descrito en $\mathrm{CRMO}^{1}$.

Tabla 3. Predictores de enfermedad crónica en osteomielitis crónica multifocal recurrente.

\begin{tabular}{c}
\hline Predictor \\
\hline Género femenino \\
Compromiso de la pared anterior del tórax \\
Artritis periférica \\
Lesiones en piel \\
Elevación de los reactantes de fase aguda
\end{tabular}

Se han descrito casos en los que la CRMO evoluciona a una espondiloartritis, con reportes de niños con este diagnóstico que desarrollan entesitis y artritis psoriásica, a pesar de recibir manejo adecuado $8,50,51$.

Respecto a las secuelas a largo plazo, hasta una cuarta parte de los pacientes continúa experimentando dolor crónico luego de años de seguimiento, relacionado con la presencia de deformidades residuales. El compromiso de la columna vertebral implica riesgo de fracturas por compresión o colapso vertebral con el posterior desarrollo de cifosis, escoliosis o compresión espinal ${ }^{51}$. En algunos niños, el compromiso de la placa fisiaria lleva a un cierre prematuro y causa discrepancia en la longitud de extremidades o deformidades óseas ${ }^{1}$.

\section{CONCLUSIONES}

La osteomielitis crónica multifocal recurrente es una enfermedad autoinflamatoria caracterizada por focos estériles de inflamación ósea que producen dolor recurrente. Su presentación clínica también involucra lesiones en piel que lo ubican dentro del espectro del síndrome: artritisdermatitis con el síndrome Sapho. Para su diagnóstico se requiere un alto índice de sospecha especialmente por el amplio diagnóstico que incluye infecciones y tumores óseos. El tratamiento consiste en el uso de antiinflamatorios no esteroideos. El pronóstico de esta enfermedad, en general, es bueno, con un curso clínico benigno y autolimitado en la mayoría de los casos.

\section{DECLARACIÓN \\ DE CONFLICTO DE INTERESES}

Los autores declaran no tener ningún conflicto de interés durante el proceso de elaboración de este manuscrito. La revisión fue financiada con recursos propios.

\section{REFERENCIAS}

1. Costa-Reis P, Sullivan K. Chronic recurrent multifocal osteomyelitis. J Clin Immunol. 2013; 33: 1043-1056.

2. Wipff J, Adamsbaum C, Kahan A, Job-Deslandre C. Chronic recurrent multifocal osteomielitis. Joint Bone Spine. 2011; 78: 555-560.

3. Schultz C, Holterhus PM, Seidel A. Chronic recurrent multifocal osteomyelitis in children. Pediatric Infect Dis J. 1999; 18: 1008-1013.

4. Aelami M, Ahanchian H, Esfehani RJ. Chronic recurrent multifocal osteomyelitis; manifestation, imaging and diagnosis. Iran J Nucl Med. 2014; 22: 29-32.

5. Giedion A, Holthusen W, Masel LF, Vischer D. Subacute and chronic "symmetrical" osteomyelitis. Ann Radiol (Paris). 1972; 15: 329-342.

6. Rosenberg ZS, Shankman S, Klein M, Lehman W. Chronic Recurrent Multifocal Osteomyelitis. AJR. 1988; 151: 142-144.

7. Björkstén B, Gustavson $\mathrm{KH}$, Eriksson B, Lindholm A, Nordström S. Chronic recurrent multifocal osteomyelitis and pustulosis palmoplantaris. J Pediatr. 1978; 93: 227-231. 
8. Nguyen MT, Borchers A, Selmi C, Naguwa SM, Cheema G, Gershwin ME. The SAPHO syndrome. Semin Arthritis Rheum. 2012; 42: 254-265.

9. Carneiro S, Sampaio-Barros PD. SAPHO Syndrome. Rheum Dis Clin N Am. 2013; 39: 401-418.

10. Aygun D, Barut K, Camcioglu Y, Kasapcopur O. Chronic recurrent multifocal osteomyelitis: A rare skeletal disorder. BMJ Case Rep. 2015; pii: bcr2015210061.

11. Surendra G, Shetty U. Chronic recurrent multifocal osteomyelitis: A rare entity. J Med Imaging Radiat Oncol. 2015; 59: 436-444.

12. Iyer R, Thapa M, Chew F. Chronic Recurrent Multifocal Osteomyelitis: Review. AJR 2011; 196: S87-91.

13. Assmann $G$, Kueck $O$, Kirchhoff $T$, Rosenthal $H$, Voswinkel J, Pfreundschuh $M$, et al. Efficacy of antibiotic therapy for SAPHO syndrome is lost after its discontinuation: an interventional study. Arthritis Res Ther. 2009; 1: R140.

14. Girschick HJ, Huppertz HI, Harmsen D, Krauspe R, Müller-Hermelink HK, Papadopoulos T. Chronic recurrent multifocal osteomielitis in children: diagnostic value of histopathology and microbial testing. Hum Pathol. 1999; 30: 59-65.

15. Job-Deslandre C, Krebs S, Kahan A. Chronic recurrent multifocal osteomielitis: five-year outcomes in 14 pediatric cases. Joint Bone Spine. 2001; 68: 245-251.

16. Grosjean C, Hurtado-Nedelec M, Nicaise-Roland P, Ferreyra-Dillon R, Bollet C, Quintin E, et al. Prevalence of autoantibodies in SAPHO syndrome: a single-center study of 90 patients. J Rheumatol. 2010; 37: 639-643.

17. Roderick MR, Ramanan AV. Chronic recurrent multifocal osteomielitis. Adv Exp Med Biol. 2013; 764: 99-107.

18. Barral E, Freire $X$, Enriquez E, Casado R, Bello P, de Inocencio J. Non-bacterial chronic osteomyelitis: Experience in a tertiary hospital. An Pediatr (Barc). 2015; pii: S16954033(15)00354-9.

19. Johnsson A, Flatø B, Knudsen PK, Lilleby V. Clinical outcome in a Norwegian cohort of patients with chronic recurrent multifocal osteomyelitis. Scand J Rheumatol. 2015; 44: 513-514.

20. Monsour PA, Dalton JB. Chronic recurrent multifocal osteomielitis involving the mandible: case reports and review of the literature. Dentomaxillofac Radiol. 2010; 39: 184-190.

21. ShivShanker V, Paterson JM, Dodd SJ. Hypertrophic osteitis of the medial end of the clavicle. Pediatr Orthop B. 1999; 8: 48-49.

22. Falip C, Alison M, Boutry N, Job-Deslandre C, Cotten A, Azoulay R, et al. Chronic recurrent multifocal osteomielitis (CRMO): a longitudinal case series review. Pediatr Radiol 2013; 43: 355-375.

23. Kaiser D, Bolt I, Hofer M, Relly C, Berthet G, Bolz D, et al. Chronic nonbacterial osteomyelitis in children: a retrospective multicenter study. Pediatr Rheumatol Online J. 2015; 13: 25.

24. Walsh P, Manners PJ, Vercoe J, Burgner D, Murray KJ. Chronic recurrent multifocal osteomyelitis in children: nine years' experience at a statewide tertiary paediatric rheumatology referral centre. Rheumatology. 2015; 54: 1688-1691.

25. Bousvaros A, Marcon M, Treem W, Waters $P$, Issenman $R$, Couper $R$, et al. Chronic recurrent multifocal osteomielitis associated with chronic inflammatory bowel disease in children. Dig Dis Sci. 1999; 44: 2500-2507.

26. Williamson $D$, Sibbald RG. Chronic recurrent multifocal osteomielitis: a rare association with pyoderma gangrenosum in adults. Br J Dermatol. 2002; 147: 611-613.

27. Jansson A, Renner ED, Ramser J, Mayer A, Haban M, Meindl A, et al. Classification of nonbacterial osteitis: retrospective study of clinical, immunological and genetic aspects in 89 patients. Rheumatology. 2007; 46: 154-160.

28. Jansson AF, Müller TH, Gliera L, Ankerst DP, Wintergerst U, Belohradsky BH, et al. Clinical score for nonbacterial osteitis in children and adults. Arthritis Rheum. 2009; 60: 1152-1159.

29. Depasquale R, Kumar N, Lalam RK, Tins BJ, Tyrrell PN, Singh J, et al. SAPHO: What radiologists should know. Clin Radiol 2012;67:195-206.

30. Thakur U, Blacksin M, Beebe K. Synovitis, Acne, Pustulosis, Hyperostosis and Osteitis (SAPHO) and chronic recurrent multifocal osteomyelitis (CRMO): Role of imaging in diagnosis. Radiography 2012; 18: 221-224.

31. Tan BS, Nayanar V, Mansberg R, Murray IP, Rossleig MA. Two cases of chronic recurrent multifocal ostomyelitis: Radiological and scintigraphic findings. Australas Radiol 1996; 40: 437-441.

32. Jurik AG, Graudal $H$, de Carvalho A. Sclerotic changes of the manubrium sterni. Skeletal Radiol. 1985; 13: 195-201.

33. Jurik AG, Egund N. MRI in chronic recurrent multifocal osteomielitis. Skeletal Radiol. 1997; 26: $230-238$.

34. Guérin-Pfyffer S, Guillaume-Czitrom S, Tammam S, Koné-Paut I. Evaluation of chronic recurrent multifocal osteitis in children by whole-body magnetic resonance imaging. Joint Bone Spine. 2012; 79: 616-620.

35. Fritz J. The Contributions of Whole-body Magnetic Resonance Imaging for the Diagnosis and Management of Chronic Recurrent Multifocal Osteomyelitis. J Rheumatol. 2015; 42: 1359-1360.

36. Voit AM, Arnoldi AP, Douis H, Bleisteiner F, Jansson MK, Reiser MF et al. Whole-body Magnetic Resonance Imaging in Chronic Recurrent Multifocal Osteomyelitis: Clinical Longterm Assessment May Underestimate Activity. J Rheumatol. 2015; 42: 1455-1462. 
37. Mandell GA, Contreras SJ, Conard K, et al. Bone scintigraphy in the detection of chronic recurrent multifocal osteomielitis. J Nucl Med 1998; 39: 1778-83.

38. Fritz J, Tzaribachev N, Thomas C. Magnetic resonance imaging-guided osseous biopsy in children with chronic recurrent multifocal osteomielitis. Cardiovasc Intervent Radiol. 2012; 35: 146-153.

39. Jurik AG. Chronic recurrent multifocal osteomyelitis. Semin Musculoskelet Radiol. 2004; 8: 243-253.

40. Kundu BK, Naik AK, Bhargava S, et al. Diagnosing the SAPHO syndrome: a report of three cases and review of literature. Clin Rheumatol. 2013; 32: 1237-1243.

41. Tlougan BE, Podjasek JO, O'Haver J. Chronic recurrent multifocal osteomielitis (CRMO) and synovitis, acne, pustulosis, hyperostosis, and osteitis (SAPHO) síndrome with associated neutrophilic dermatoses: a report of seven cases and review of the literature.Pediatr Dermatol. 2009; 26: 497-505.

42. Holden W, David J. Chronic recurrent multifocal osteomyelitis: two cases of sacral disease responsive to corticosteroids.Clin Infect Dis. 2005; 40: 616-619.

43. Handly B, Moore M, Creutzberg G, Groh B, Mosher T. Bisphosphonate therapy for chronic recurrent multifocal osteomyelitis. Skeletal Radiology 2013;42: 1741-2.

44. Hospach $T$, Langendoerfer $M$, von Kalle T, Maier J, Dannecker GE. Spinal involvement in chronic recurrent multifocal osteomielitis (CRMO) in childhood and effect of pamidronate. Eur J Pediatr. 2010; 169: 1105-1111.

45. Gleeson H, Wiltshire E, Briody J, Hall J, Chaitow J, Sillence D, et al. Childhood chronic recurrent multifocal osteomyelitis: pamidronate therapy decreases pain and improves vertebral shape. J Rheumatol. 2008; 35: 707-712.

46. Marangoni RG, Halpern AS. Chronic recurrent multifocal osteomielitis primarily affecting the spine treated with anti-TNF therapy. Spine (Phila Pa 1976). 2010; 35: E253-6.

47. Eisenstein EM, Syverson GD, Vora SS, Williams CB. Combination therapy with methotrexate and etanercept for refractory chronic recurrent multifocal osteomyelitis. J Rheumatol. 2011; 38: 782-783.

48. Batu ED, Ergen FB, Gulhan B, Topaloglu R, Aydingoz U, Ozen S. Etanercept treatment in five cases of refractory chronic recurrent multifocal osteomyelitis (CRMO). Joint Bone Spine. 2015; 82: 471-473.

49. Andersson R. Effective treatment with interferon-alpha in chronic recurrent multifocal osteomyelitis. J Interferon Cytokine Res. 1995; 15: 837-838.

50. Schilling F, Fedlmeier M, Eckardt A. Vertebral manifestation of chronic recurrent multifocal osteomyelitis. Rofo. 2002; 174: 1236-1242.

51. Deogaonkar K, Ghandour A, Jones A. Chronic recurrent multifocal osteomielitis presenting as acute scoliosis: A case report and review of literature. Eur Spine J. 2008; 17: S248-252. 